\title{
Riesgo nutricional en pacientes con tuberculosis pulmonar: ¿cuestión del paciente o de los servicios de salud?
}

\author{
Georgina Mayela N úñez-Rocha, M.C ., M.S.P., ${ }^{(1)}$ A na María Salinas-Martínez, M.C ., D r. en S. P., ${ }^{(1,2)}$ \\ Enrique Villarreal-Ríos, M. C., M.C.S.S., ${ }^{(1)}$ Ma. Eugenia Garza-Elizondo, Lic. en Enf., ${ }^{(1)}$ \\ Francisco González-Rodríguez, M.C., M.S.P. ${ }^{(2)}$
}

\begin{abstract}
Núñez-Rocha GM, Salinas-Martínez AM, Villarreal-Ríos E, Garza-Elizondo ME, González-Rodríguez F. Riesgo nutricional en pacientes con tuberculosis pulmonar: ¿cuestión del paciente o de los servicios de salud? Salud Publica Mex 2000;42:126-132.
\end{abstract}

\section{Resumen}

Objetivo. Identificar factores de riesgo nutricional en pacientes con tuberculosis pulmonar (TBP). Material y métodos. Se seleccionaron al azar 185 pacientes con TBP atendidos en dos instituciones de salud de Monterrey, $\mathrm{N}$ uevo León, México, durante 1997. Se identificaron variables antropométricas, socioeconómicas, utilización del servicio de nutrición, accesibilidad a los alimentos, efectos secundarios de drogas antifímicas, y atribución de la enfermedad a la alimentación. El plan de análisis incluyó estadística descriptiva, análisis bivariado y multivariado de regresión lo gística múltiple, además se estimó razón de prevalencia e intervalos de confianza de $95 \%$. Resultados El promedio de edad fue de $42.4 \pm 19.9$ años. La media de índice de masa corporal fue de $19.8 \pm 3.2$ y se encontraban desnutridos $56.8 \%$ del total de los pacientes. El $26.4 \%$ de éstos fue enviado al servicio de nutrición y, únicamente, $24.3 \%$ lo utilizó. El análisis multivariado mostró como factores de riesgo para desnutrición a los efectos secundarios de las drogas antifímicas, independientemente de la edad, sexo, escolaridad, ocupación, tiempo de evolución, accesibilidad a los alimentos, atribución de la enfermedad al tipo de alimentación y utilización del servicio de nutrición $\left(\chi^{2}=10.58 ; p=0.0515\right.$, $\left.r^{2}=0.42\right)$. Conclusiones El riesgo nutricional al que se en-

\author{
Núñez-Rocha GM, Salinas-Martínez AM, \\ Villarreal-Ríos E, Garza-Elizondo ME, \\ González-Rodríguez F. \\ Nutritional risk in patients \\ with pulmonary tuberculosis. A patient \\ or a health services issue? \\ Salud Publica Mex 2000;42:126-132.
}

\begin{abstract}
A bstract
Objective. To determine nutritional risk factors in a po pulation of patients with pulmonary tuberculosis (PT). Material and methods. During 1997, one-hundred and eighty-five patients with PT were chosen at random from two health institutions in Monterrey, N uevo León, México. Variables analyzed were: anthropometric measures, socioecono mic status, utilization of the nutrition clinic, access to foodstuffs, adverse reactions to drugs, and disease attributable to malnutrition. Statistical analysis consisted of descriptive, bivariate, and multivariate logistic regression, in addition to prevalence ratios and $95 \%$ confidence intervals. Results. The mean age was $42.4 \pm 19.9$ years. The mean body mass index was $19.8 \pm 3.2 ; 57 \%$ of patients presented malnutrition; $26 \%$ of them were referred to the nutrition clinic, $24.3 \%$ of whom actually attended it. Multivariate analysis showed that adverse reactions of tuberculosis drugs were risk factors for malnutrition, independent of age, gender, education, occupation, year of diagnosis and access to foodstuffs, disease atributable, and utilization of the nutrition clinic. $\left(\chi^{2}=10.58 ; p=0.051, R^{2}=0.42\right)$. Conclusions. $\mathrm{N}$ utritional risk in patients with pulmonary tuberculosis is both a patient and a health services issue. The high prevalence of malnutrition, the low utilization rate of nutritional
\end{abstract}

(1) Unidad Regional de Investigación Epidemiológica y en Servicios de Salud, Instituto Mexicano del Seguro Social, Monterrey, N uevo León, México.

(2) Departamento de Medicina Preventiva, Facultad de Medicina, Universidad Autónoma de N uevo León, Monterrey, N uevo León, México.

Fecha de recibido: 15 de noviembre de 1999 - Fecha de aprobado: 14 de enero de 2000 Solicitud de sobretiros: D ra. G eorgina Mayela N úñez Rocha. Enebro 4317, colonia Residencial Cedros, 64370 Monterrey, N uevo León, México. Correo electrónico: imssnorteinv@ infosel.net.mx 
frenta el paciente es responsabilidad tanto de éste, por la escasa utilización que hace del servicio de nutrición, como de los servicios de salud, debido a la existencia de barreras de tipo organizacional que dificultan el acceso al servicio de nutrición. A demás, la falta de accesibilidad a los alimentos y el impacto de los efectos secundarios de las drogas antifímicas justifican la necesidad de focalizar la atención en este grupo de riesgo.

Palabras clave: nutrición; factores de riesgo; tuberculosis pulmonar; México services, and the effect of adverse reactions to therapeutic drugs, justify the need to focus attention on this particular group of patients.

Key words: nutrition; risk factors; tuberculosis, pulmonary; Mexico
$\mathrm{L}$ a tuberculosis pulmonar (TBP) es un problema de salud pública de gran magnitud. Se estima en el mundo un total de 90 millones de casos nuevos y 30 millones de muertes por esta causa para el año 2000. Se considera que el sudeste asiático y África son las zonas de mayor riesgo. La tasa de prevalencia en el ámbito mundial es de 61.8/100 000 habitantes y en América de 34.2. ${ }^{1}$ En la República mexicana la mortalidad por enfermedades trasmisibles se concentra en áreas de muy alta marginación y en especial la tuberculosis pulmonar. La tasa de mortalidad que se observó fue de 5.8/100 000 en el ámbito nacional, y en Nuevo León, de 6.2/100 000,² en 1992.

La TBP y sus causas multifactoriales obligan a abordar el problema de manera integral, pues el difícil control de este padecimiento hace imprescindible la vigilancia por un equipo multidisciplinario de salud para incidir sobre este proceso de salud-enfermedad. ${ }^{3}$ En la actualidad el aspecto nutricional de los pacientes con TBP ha estado relegado, sin embargo, la nutrición debe ocupar un lugar prioritario desde el punto de vista preventivo. El presente estudio así lo considera con la finalidad de coadyuvar a la solución de este problema desde su raíz. Se sabe que $90 \%$ de los estados de malnutrición en nuestro medio son ocasionados por una sola causa, la alimentación inadecuada, en términos de alimentación pobre o excesiva, determinada por la ignorancia, la accesibilidad que se tenga a los alimentos, el hambre, la falta de higiene o bien por alteraciones en la calidad y la cantidad de los alimentos consumidos, ${ }^{4}$ entre otros factores.

La relación entre desnutrición y TBP es interactiva porque la desnutrición prolongada expone al organismo a una invasión fácil de enfermedades infectocontagiosas y la infección por TBP conduce o agrava la desnutrición. 5,6 La trascendencia de encontrarse en un estado nutricional inadecuado es porque se desencadenarían trastornos en muchas de las funciones del organismo, como las inmunológicas. ${ }^{7,8}$

En los pacientes que ingieren drogas antifímicas se ha demostrado que éstas actúan provocando trastornos gastrointestinales que alteran la absorción de nutrimentos y micronutrimentos, además de ocasionar sintomatología como náusea, vómito y alteración en los sentidos del gusto y del olfato, lo que tiene como consecuencia la disminución en el consumo de alimentos por el paciente y, por ende, el deterioro del estado nutricio. $^{9}$

Otro factor que afecta la situación nutricional de estos pacientes es su condición socioeconómica, fundamentalmente por dos razones: una, el ingreso familiar para acceder al consumo de alimentos y, dos, un determinado nivel educativo que permite a la población orientar su consumo, optimizando los recursos de manera selectiva, para asignar un determinado porcentaje de su ingreso para gasto en alimentación. ${ }^{10,11}$

Es importante, además, considerar la utilización del servicio de nutrición tanto por el prestador de servicio, fundamentalmente el médico familiar, como por el mismo paciente. Por esto, el propósito del presente estudio fue identificar los factores de riesgo nutricional en los pacientes con tuberculosis pulmonar en el estado de Nuevo León.

\section{Material y métodos}

Se llevó a cabo un estudio transversal. Se seleccionaron al azar 185 pacientes, de una base de datos generada en el año de 1997, que incluía a pacientes de 12 años y más de edad, con diagnóstico de TBP (baciloscopía positiva), residentes de Monterrey y su área metropolitana, activos en el Programa de Atención y Control de la TBP en el Instituto Mexicano del Seguro Social (IMSS) y Hospital Universitario (HU), de la Fa- 
cultad de Medicina de la Universidad Autónoma de Nuevo León. Se excluyeron los pacientes con índice de masa corporal (IMC) mayor a $25.0 \mathrm{~kg} / \mathrm{m}^{2}$.

El cálculo del tamaño de la muestra se realizó de acuerdo con la fórmula para estimar una proporción en una población finita, ${ }^{12}$ considerando una $\mathrm{N}=251$ y una prevalencia hipotética de $40 \%$ de desnutrición en pacientes con TBP (proporción estimada en una prueba piloto). Se empleó un margen de error de $5 \%$ y un intervalo de confianza de $95 \%$. El resultado indicó que se requería un mínimo de 125 pacientes con TBP.

La base contenía datos obtenidos de un cuestionario estructurado que se aplicó mediante una entrevista directa en el hogar del paciente: de éstos, $50.8 \%$ fue diagnosticado en el mismo año de la encuesta; $31.1 \%$, en el año anterior a la encuesta, y el resto, dos años antes de la encuesta. Dicha base incluía información de las características demográficas, como edad y sexo; socioeconómicas, como ingreso familiar, ocupación y escolaridad; además, se les interrogó acerca de la proporción del ingreso semanal gastado en alimentación con el fin de determinar la accesibilidad a los alimentos, de acuerdo con los indicadores que establecen la falta de acceso cuando una familia gasta más de $50 \%$ de su ingreso en la compra de alimentos. ${ }^{13}$ Para determinar la utilización del servicio de nutrición por parte del paciente y del prestador de servicio, se les cuestionó acerca del número de veces que había sido enviado por el médico de consulta externa de medicina familiar al servicio de nutrición y dietética y cuántas de ellas el paciente había acudido a consulta. Contenía también una pregunta acerca de la atribución del paciente hacia la enfermedad con relación al tipo de alimentación y a los efectos secundarios de las drogas antifímicas que presentara el paciente, tales como anorexia, náusea, mareo, vómito, dolor abdominal y alteración en el sabor y el olor de los alimentos.

Asimismo, antes de efectuar la visita al domicilio del paciente, se capacitó a los examinadores con relación a los métodos, técnicas y procedimientos que se emplean con la finalidad de estandarizar criterios para la toma de medidas antropométricas, de acuerdo con lo recomendado por Habicht ${ }^{14}$ y las especificaciones establecidas por Lohman y colaboradores ${ }^{15}$ con el fin de recolectar los datos que permitieran su replicabilidad, y minimizaran los errores entre las distintas mediciones. Las medidas antropométricas fueron colectadas por dos personas, una de ellas tomó el peso y la talla del paciente, y la otra anotó los datos. Para determinarlas se utilizó la báscula con estadímetro en centímetros y precisión de hasta $100 \mathrm{mg}$ con balance máximo de 140 kilogramos y a partir de ellas se estimó el índice de masa corporal (IMC) o índice de Quetelet, que incluye en la fórmula el peso en kilogramos sobre la talla en metros al cuadrado. Se consideró desnutrición cuando los indicadores se ubicaron en 20 puntos o menos del IMC y como normal de 20.1 a $24.9 .{ }^{16} \mathrm{El}$ plan de análisis incluyó estadística descriptiva, análisis bivariado y se elaboró un modelo de regresión logística múltiple, con el fin de aislar cada uno de los factores que intervinieron en el estado nutricional. Además se estimaron razón de prevalencias (RP) e intervalos de confianza(IC) de $95 \%$.

\section{Resultados}

El promedio de edad de los pacientes fue de $42.4 \pm 19.9$ años, de éstos $53 \%$ eran de sexo masculino, $63 \%$ estaba casado o en unión libre, y en cuanto a la escolaridad, $57.6 \%$ tenía primaria o no tenía estudios. El $32.6 \%$ se dedicaba a labores del hogar. El ingreso familiar semanal era de $568.87 \pm 464.82$ pesos, los pacientes predominantemente pertenecían al IMSS (91.3\%). En el cuadro I se pueden observar más detalles sociodemográficos.

La historia de síntomas mostró a la tos con sus diferentes variantes como la manifestación clínica predominante, habiendo presentado una media de $3.6 \pm 8.6$ meses y una mediana de 1.0, desde el inicio de los mismos hasta la fecha del diagnóstico.

La rifampicina fue la droga antifímica más comúnmente indicada, seguida por la combinación de isoniacida, rifampicina y pirazinamida. En el cuadro II se muestra con detalle la utilización de drogas antifímicas y el tiempo de administración correspondiente. El $64.6 \%$ de los entrevistados presentó algún tipo de efecto secundario: náuseas, $27.3 \%$; vómito, $17.6 \%$; mareo, $24 \%$; dolor abdominal, $24 \%$; falta de hambre, $27.6 \%$, y cambio en el sabor u olor de los alimentos, $22.4 \%$.

El 43.2\% atribuyó el desarrollo de la enfermedad a su tipo de alimentación. La media del IMC fue de $19.8 \pm 3.2 ; 31.9 \%$ presentó muy bajo peso; $24.9 \%$, bajo peso, y $43.2 \%$, peso normal. El $26.4 \%$ de los pacientes entrevistados refirió haber sido enviado al departamento de nutrición. La tasa de utilización del servicio de nutrición y dietética por parte de los pacientes referidos fue de $24.3 \%$.

En cuanto al análisis bivariado, en donde se analizaron los factores de riesgo para estado nutricional, sociodemográficos y los referentes a la alimentación, solamente la accesibilidad a los alimentos mostró una asociación estadísticamente significativa con desnutrición (cuadro III).

El análisis multivariado mostró como factores de riesgo para desnutrición, a los efectos secundarios de las drogas antifímicas, no así, las variables edad, 
Cuadro I

Características sociodemográficas

DE 185 PACIENTES CON TUBERCULOSIS PULMONAR. Monterrey, Nuevo León, 1997

\begin{tabular}{|c|c|}
\hline Característica & $\%$ \\
\hline \multicolumn{2}{|l|}{ Edad (años cumplidos) } \\
\hline $12-15$ & 2.7 \\
\hline $16-25$ & 23.8 \\
\hline $26-35$ & 19.5 \\
\hline $36-45$ & 12.4 \\
\hline $46-55$ & 11.9 \\
\hline $56-65$ & 13.5 \\
\hline $66+$ & 16.2 \\
\hline \multicolumn{2}{|l|}{ Sexo } \\
\hline Masculino & 53.0 \\
\hline Femenino & 47.0 \\
\hline \multicolumn{2}{|l|}{ Estado Civil } \\
\hline Soltero & 27.6 \\
\hline Casado y unión libre & 63.0 \\
\hline 0 tro & 9.4 \\
\hline \multicolumn{2}{|l|}{ Escolaridad } \\
\hline Primaria o menos & 57.6 \\
\hline Secundaria & 25.0 \\
\hline Preparatoria & 10.9 \\
\hline Profesional & 6.5 \\
\hline \multicolumn{2}{|l|}{ O cupación } \\
\hline Labores del hogar & 32.6 \\
\hline Empleado & 20.5 \\
\hline O brero & 16.0 \\
\hline Desempleado & 22.1 \\
\hline Estudiante & 6.6 \\
\hline Jubilado o pensionado & 2.2 \\
\hline \multicolumn{2}{|l|}{ Institución de salud } \\
\hline Instituto Mexicano del Seguro Social & 91.3 \\
\hline Hospital Universitario & 8.7 \\
\hline
\end{tabular}

sexo, escolaridad, ocupación, tiempo de evolución, accesibilidad a los alimentos, atribución de la enfermedad al tipo de alimentación y utilización del servicio de nutrición $\left(\chi^{2}=10.58 ; p=0.0515 ; r^{2}=0.42\right)$ (cuadro IV).

\section{Discusión}

El promedio de edad en la que se presentó el problema en estos pacientes fue precisamente en la etapa productiva, gran parte de ellos eran casados y con un ingreso familiar bajo; esto permitió estimar su poten-

\section{Cuadro II \\ Utilización de dROGas ANTIFÍMiCAS de 185 PACIENTES CON tUberculosis PUlmonar. Monterrey, NueVo LEÓN, 1997*}

Frecuencia $\begin{gathered}\text { Tiempo de adminis- } \\ \text { tración al momento } \\ \text { de la encuesta }\end{gathered}$
$\begin{aligned} & \text { (\%) } \begin{array}{c}\text { Promedio Desviación } \\ \text { (meses) estándar }\end{array}\end{aligned}$

\begin{tabular}{lrrr} 
Isoniacida, rifampicina y pirazinamida & 37.2 & 6.8 & 7.1 \\
\hline Rifampicina e isoniacida & 16.9 & 3.4 & 2.4 \\
\hline Rifampicina & 46.8 & 6.8 & 11.8 \\
\hline Isoniacida & 22.2 & 8.0 & 11.0 \\
\hline Ethambutol & 24.0 & 12.1 & 17.4 \\
\hline Pirazinamida & 16.4 & 10.4 & 14.9 \\
\hline Estreptomicina & 11.7 & 1.7 & 1.2
\end{tabular}

* Más de una droga antifímica pudo haber sido utilizada por el mismo paciente

cial de compra de alimentos e identificar a un grupo en el cual el proceso salud-enfermedad es de gran trascendencia, sobre todo porque incluye en su mayoría a población cubierta por la seguridad social y se trata de un grupo que interviene directamente en el sector productivo del país. ${ }^{17}$ Sin embargo, hubiera sido importante analizar a una mayor proporción de población abierta que acude a unidades del sector público y no tiene las mismas características que la de seguridad social, esto nos da pauta para futuros estudios.

La prevalencia de desnutrición en esta población fue de $56.8 \%$, cifra más elevada a la estimada en un estudio realizado en el estado de Veracruz, que fue de $36.4 \%$, aunque a diferencia del presente trabajo, los datos correspondían a personas que habían fallecido por TBP; ${ }^{18}$ y menor a la establecida en una población de Tanzania, en donde se estudiaron 148 pacientes antes del tratamiento antifímico, de éstos, $77 \%$ de los varones y $58 \%$ de las mujeres tenían un IMC $<18.5$ y $20 \%$ de todos ellos tenían un IMC <16.0; lo que pone de manifiesto el riesgo de padecer desnutrición y su asociación con infecciones ${ }^{19}$. Esto permite observar la interacción que existe entre el estado de nutrición y la TBP. Aunque es importante aclarar que el presente estudio se realizó en población con diagnóstico de TBP sin otras enfermedades concomitantes y con terapéutica medicamentosa ya establecida.

La falta de acceso a la alimentación es un factor predictivo de serios problemas en el estado nutricio del paciente. Esta situación refleja el desarrollo interdependiente y desigual de algunos sectores de la 


\section{Cuadro III \\ FACTORES de RIESGo de DESNUTRICIÓN EN 185 PACIENTES CON TUBERCULOSIS PULMONAR. MonterRey, Nuevo León, 1997}

\begin{tabular}{|c|c|c|c|c|c|}
\hline \multirow[t]{2}{*}{ Factor de riesgo } & \multicolumn{2}{|c|}{ Desnutrido } & \multirow[b]{2}{*}{ RP } & \multirow[b]{2}{*}{ IC $95 \%$} & \multirow[b]{2}{*}{$p$} \\
\hline & Sí & No & & & \\
\hline Accesibilidad a los alimentos & $n=105$ & $n=80$ & & & \\
\hline Gasto de $49.9 \%$ o menos del ingreso familiar en alimentación & $22(21)$ & $6(7.5)$ & 1.00 & & \\
\hline Gasto de $50 \%$ o más del ingreso familiar en alimentación & $83(79)$ & $74(92.5)$ & 0.31 & $0.10-0.85$ & 0.0202 \\
\hline Atribución de la enfermedad a tipo de alimentación & $n=10$ & $n=39$ & & & \\
\hline Sí & $5(50)$ & $30(77)$ & 1.00 & & \\
\hline No & $5(50)$ & $9(23)$ & 0.30 & $0.06-1.57$ & 0.1973 \\
\hline Utilización del servicio de nutrición y dietética por el paciente & $n=8$ & $n=34$ & & & \\
\hline Sí & $4(50)$ & $24(70.5)$ & 1.00 & & \\
\hline No & $4(50)$ & $10(29.5)$ & 0.42 & $0.07-2.54$ & 0.4872 \\
\hline Uno o más efectos secundarios a drogas antifímicas & $n=104$ & $n=77$ & & & \\
\hline Sí & $71(68.3)$ & $46(59.7)$ & 1.45 & $0.75-2.81$ & 0.3032 \\
\hline No & $33(31.7)$ & $31(40.3)$ & 1.00 & & \\
\hline
\end{tabular}

RP: razón de prevalencias

IC : intervalo de confianza

población como sería el caso de este grupo, en el cual se encuentran limitados los recursos para satisfacer adecuadamente una de las necesidades primarias del ser humano, como es la alimentación; situación de-

\section{Cuadro IV \\ Modelo de RegResión logística MúltiPLE EN 110 PACIENTES CON TUBERCULOSIS PULMONAR. Monterrey, Nuevo León , 1997}

\begin{tabular}{lcccc} 
Factor & Coeficiente & RP & IC95\% & $p$ \\
Edad & -0.70 & .98 & $.74-1.3$ & 0.945 \\
\hline Sexo & -.923 & 2.1 & $.78-6.0$ & 0.133 \\
\hline Escolaridad & .237 & 1.0 & $.65-1.7$ & 0.813 \\
\hline Ocupación & 1.51 & .89 & $.63-1.2$ & 0.502 \\
\hline Tiempo de evolución & -0.92 & .84 & $.57-1.2$ & 0.358 \\
\hline Accesibilidad a los alimentos & -1.51 & .99 & $.99-1.0$ & 0.211 \\
\hline Atribución de la enfermedad al tipo & & & & \\
de alimentación & -1.29 & .11 & $.72-1.27$ & 0.738 \\
\hline Utilización del servicio de nutrición & & & & \\
y dietética por el paciente & 2.29 & .69 & $.27-1.7$ & 0.947 \\
\hline Efectos secundarios a drogas antifímicas & 1.78 & 3.3 & $1.1-9.6$ & 0.024 \\
$\chi^{2}=1$ 058; $p=0.0515 ; r^{2}=0.42$ & & & & \\
RP: razón de prevalencias & & & & \\
IC: intervalo de confianza & & & &
\end{tabular}

rivada del sistema político, económico y social en el que se está inmerso. ${ }^{10,11}$ En este grupo se observó que cuando se gasta una mayor proporción del ingreso en alimentación, menor es el riesgo de padecer desnutrición, no obstante, este efecto desapareció en el análisis multivariado. En otros países se ha tomado la decisión de proporcionar ayuda alimentaria directa durante el tratamiento antifímico, y se ha visto que la recuperación es más rápida. ${ }^{20}$

Por otro lado, con relación a la atribución de la enfermedad a problemas de alimentación y considerando lo que supone dicha teoría, ${ }^{21}$ se observa que en este estudio no tuvo significancia estadística; no por eso se debe dejar de lado, pues se percibe la falta de conocimiento que existe en la población estudiada, y esto sólo indica que el paciente no se encuentra lo suficientemente enterado, específicamente en el aspecto nutricional y, por tanto, no atribuye a la alimentación el deterioro o la dificultad en el control de su padecimiento. ${ }^{22}$

Otro aspecto importante de este estudio fue la escasa utilización del servicio de nutrición y dietética por el paciente; este hecho puede explicarse porque el personal de salud no lo tiene en su esquema de tratamiento, ya que en la norma oficial no se contempla el envío a este servicio y, aún peor, se considera a la dieta 
del paciente como un factor "no importante" dentro de los que influyen en la respuesta al tratamiento; ${ }^{23-25}$ a pesar de que en algunos estudios se ha demostrado que un estado nutricio adecuado se asocia con una mayor sobrevida y también disminuye el tiempo para que el paciente llegue a una etapa de curación. . $^{2026-28}$ Otra explicación puede ser que a pesar de las intenciones del médico para enviar a estos pacientes al servicio de nutrición, se encuentra con barreras de acceso al servicio sobre todo de tipo organizacional, como es el hecho de depender de trámites hasta cierto punto burocráticos para solicitar cita con el especialista en nutrición, y sea este factor el que intervenga en dicho personal para que no utilice este servicio y, por lo tanto, el paciente, aún menos. En el presente estudio no influyó el hecho de utilizar o no el servicio de nutrición y dietética sobre el estado nutricional, debido probablemente al escaso número de pacientes que se habían enviado y que se analizaron. Aunque el hecho de que el médico derive al paciente al departamento de nutrición no indica precisamente que él lo vaya a utilizar. En este trabajo, además de que no tenía la información apropiada, el paciente escasamente acudía a solicitar atención, y no tenía, además, la conciencia de que si se encontrara con un estado nutricional adecuado, coadyuvaría a la mejoría y curación de su padecimiento.

En este estudio, la ingestión de drogas antifímicas jugó un papel muy importante en función de los efectos secundarios que éstas provocaron en el aparato digestivo, y que se asociaron con desnutrición, como se estableció en el modelo de regresión logística, mediante el cual, al aislar cada uno de los factores, el referido fue el único significativo. Sin embargo, la reducción en el número de observaciones por valores faltantes, pudo afectar los resultados del resto de los factores que participaron en dicho modelo. Es en este aspecto, cuando el riesgo es responsabilidad de los servicios de salud, porque es evidente que se debe considerar el estado nutricional de estos pacientes antes y durante la terapia antifímica, pues se ha establecido que pacientes con tuberculosis pulmonar han fallecido en estado de emaciación y con serios problemas nutrimentales a nivel celular, este hecho eventualmente podría afectar la inmunidad e intervenir en el proceso de curación en este grupo. ${ }^{27,29,30}$

Otro punto de gran trascendencia que se ha comentado en muchos trabajos y que no fue el objetivo del presente estudio, es el alto índice de abandono de la terapéutica medicamentosa; muchas veces por los problemas que ocasionan las drogas antifímicas en su aparato digestivo, sobre todo cuando tienen alteraciones en los sentidos del gusto y el olfato, además de náusea y vómito, lo que origina una escasa adherencia al tratamiento que podría llegar al abandono, así como observaron Bustamante y colaboradores, ${ }^{18}$ y que en el presente trabajo influyeron en el estado nutricio de los pacientes con TBP. Esto de manera independiente de la información que el paciente debiera tener en cuanto al horario de ingesta de sus medicamentos, considerando desde el punto de vista clínico la biodisponibilidad de los mismos y la utilización de los nutrimentos necesarios para la conservación de un estado nutricio adecuado. ${ }^{31}$

A medida que se obtienen resultados importantes en estudios de pacientes con TBP, se necesita considerarlos para reformar las normas y los procedimientos y así monitorear, vigilar y evaluar este problema de salud en grupos de riesgo. ${ }^{32} \mathrm{Un}$ aspecto fundamental y cuya responsabilidad recae en los servicios de salud es la de asegurar el manejo apropiado, sobre todo con oportunidad; se cuenta con la infraestructura necesaria para atenderlos en el aspecto nutricional, pero se necesita una interacción más ágil del médico, del paciente y del departamento de nutrición y dietética para focalizar la atención y así establecer un flujo directo para su mejor control. Es necesario reforzar la supervisión del programa y mediante elementos de juicio proponer a las autoridades normativas cambios estructurales al mismo en donde se establezca de manera relevante el envío al servicio de nutrición. Asimismo, es conveniente instituir una red de comunicación médico-paciente-nutriólogo, para que de manera apropiada se incremente la utilización del servicio de nutrición y dietética; también es importante implantar o reforzar campañas de comunicación social para informar al paciente acerca de su padecimiento y la importancia de tener un estado nutricio adecuado. En caso necesario y con la participación del departamento de trabajo social de los servicios de salud, se podría gestionar ayuda alimentaria directa con organismos gubernamentales y no gubernamentales para los pacientes que presentaran desnutrición, por lo menos durante la aplicación del esquema terapéutico, y así puedan mejorar su estado nutricional y, por tanto, tener un mejor y más rápido control de la TBP.

En estudios posteriores, y mediante una evaluación económica, se podría determinar el impacto de estas intervenciones tanto en los servicios como en la salud de los pacientes para elaborar una propuesta concreta dirigida al sector salud.

\section{Agradecimientos}

Los autores agradecen profundamente al doctor Jesús Ancer Rodríguez, director de la Facultad de Medicina, 
de la Universidad Autónoma de Nuevo León, y al doctor José Heriberto Fabela Rodríguez, por el apoyo brindado en el desarrollo de este proyecto. Asimismo, a los estudiantes de la materia Introducción a la medicina, generación 1996-2002, por su invaluable participación.

\section{Referencias}

1. Raviglione M, Snider DE Jr, Arata K. Epidemiología glo bal de la tuberculosis. Morbilidad y mortalidad de una epidemia mundial. JAMA 1995;273: 220-226

2. Secretaría de Salud. Dirección G eneral de Estadística, Informática y Evaluación. Mortalidad 1992. México, D.F.: Secretaría de Salud, 1993.

3. Durán-González LI, Becerra-A ponte JA, Torrejón M. El proceso saludenfermedad y su interrelación con el comportamiento humano. Psicol Salud 1991;3:101-113.

4. Avila A. Hambre, desnutrición y sociedad. La investigación epidemiológica de la desnutrición en México. Guadalajara, Jalisco: Editorial U niversidad de Guadalajara, 1990.

5. Ikeogu MO, W olf B, Mathe S. Pulmonary manifestations in HIV seropositivity and malnutrition in Zimbabwe. Arch D is Child 1997;76(Feb): 124-128.

6. Manjárrez E, Serrano MV, Cano PV,Verduzco E, Escandón C, Escobedo J. Principales causas de abandono de tratamiento contra la tuberculosis pulmonar. Gac Med Mex 1993;129:57-61.

7. Handa M. A nutritional and immunological investigation of patients with tuberculosis. Kekkaku 1994;69(Jul):463-469.

8.Yoshikawa M,YonedaT, Maegawa J,T sukaguchi K,TokuyamaT, Fu A et al. Relationship between nutritional depletion and cell-mediated immune function in active pulmonary tuberculosis. Kekkaku 1994;69(A pr):307-316. 9.Zent C, Smith P. Study of the effect of concomitant food on the bioavailability of rifampicin, isoniazid and pyrazinamide. Tuber Lung $D$ is 1995; 76(A pr):109-113.

10. Cantewell MF, Mckenna M, 0 norato IM.Tuberculosis and race/ethnicity in United States. Impact of socioeconomic status. Am J Respir Crit Care Med 1998;157(Apr):1016-1020.

11. Hudelson P. Gender differentials in tuberculosis: The role of socioeconomic and cultural factors. Tuber Lung D is 1995;A pr:109-113.

12. Salinas AM, Villarreal E, Garza ME. La Investigación en ciencias de la salud, una actividad sencilla. Monterrey, N .L.: Subdirección de Educación Continua de la Facultad de Medicina, Universidad Autónoma de Nuevo León,1996.

13. Secretaría de Salubridad y Asistencia. Unidades N ormativas para la Educación en Nutrición. Dirección General de Educación para la Salud. Departamento de N utrición. México, D.F.: SSA, 1981.
14. Habicht JP. Estandarización de métodos epidemiológicos cuantitativos sobre terreno. Bol 0 ficina Sanit Panam 1974;76:375-385.

15. Lohman GT, Roche FA, Martorell R. Anthropometric standarization reference manual. C hampaing, (III): Human Kinetics Books,1988.

16. Bray GA. Complications of obesity. Ann Intern Med 1985;103:1052 1062.

17. Rojas-Soriano R. C apitalismo y enfermedad. 4a. edición. México D.F.: Plaza y Valdés editores,1988:77-145

18. Bustamante-Montes LP, Bellido-Bárcenas F, Riojas Rodríguez $\mathrm{H}$, BorjaA burto VH, Yáñez-Velasco L, Becerra-Posada F. Características sociodemográficas de personas que murieron por tuberculosis pulmonar en Veracruz, México, 1993. Salud Publica Mex 1996:38:323-331.

19. Kennedy N , Ramsay A, U iso L, G utmann J, N gowi Fl, Gillespie-SH. N utritional status and weigth gain in patients whith pulmonary tuberculosis in Tanzania. Trans R Soc Trop Med Hyg 1996;90(Mar-A pr):162-166.

20. 0 nwubalili JK. Malnutrition among tuberculosis patients in Harrow England. Eur J C lin N utr 1988;42(A pr):363-366.

21. King JB. Illness attributions and the health belief model. Health Educ $Q$ 1983;10(Fall-W inter):287-312.

22. C asas-G arcía S. Perfil sociocultural del paciente tuberculoso. Rev Med Inst Mex Seguro Soc 1996;34:229-232.

23. Manual de normas y procedimientos para la prevención y control de la tuberculosis. Grupo coordinador de programas preventivos. Sistema $\mathrm{Na}$ cional de Salud. México, D.F.: SSA, 1991:38.

24. Subdirección General Médica, Jefatura de Servicios de Atención Médica. N ormas para el manejo y control de la tuberculosis. México, D.F.: Instituto Mexicano del Seguro Social, 1989: 7-66.

25. Diario 0 ficial de la Federación, N orma 0 ficial Mexicana N O M -006SSA 2-1993 para la prevención y control de la tuberculosis en la atención primaria a la salud. México, D.F: 26 de enero de 1995:20-29.

26. Mehta JB, Fields CL, Byrd RP Jr, Roy TM. N utritional status and mortality in respiratory failure caused by tuberculosis. Tenn Med 1996; 89(0 ct): 369-371.

27.YonedaT. N utritional status and support in chronic intractable pulmonary tuberculosis. Kekkaku 1996:7(Jan):57-63.

28. Kelly P, Summerbell C. Systemic immune activation as a potential determinant of wasting in Zambians with HIV-related diarrhoea. Thorax 1996;51(Feb):132-136.

29. Amishima M, Kishi F, Kamada A, Saito N , Hamada E, Hirai $Y$ et al. Evaluation on the clinical background on early death in patients whit pulmonary during the past five years. Kekkaku 1998;73(D ec):727-731.

30. Mcmurray DN , Bartow RA, Mintzer CL, Hernández-Frontera E. Micronutrient status and immune function in tuberculosis. Ann N Y Acad Sci 1990;587:59-69.

31. Macallan DC, McN urlan MA, Kupard AV, de Souza G, Shetty PS, C alder AG et al.W hole body protein metabolism in human pulmonary tuberculosis and undernutrition: Evidence for anabolic block in tuberculosis. Clin Sci (Colch) 1998;94(Mar):321-323.

32. García-García ML,Valdespino-Gómez JL, Palacios-Martínez M, MayarMaya ME, García-Sancho C, Sepúlveda A mor J. Tuberculosis y SIDA en México. Salud Publica Mex 1995;37:539-548. 